\title{
Extended hard-X-ray emission in the inner few parsecs of the Galaxy
}

Kerstin Perez ${ }^{1,2}$, Charles J. Hailey ${ }^{1}$, Franz E. Bauer ${ }^{3,4,5}$, Roman A. Krivonos ${ }^{6}$, Kaya Mori ${ }^{1}$, Frederick K. Baganoff ${ }^{7}$, Nicolas M. Barrière $^{6}$, Steven E. Boggs ${ }^{6}$, Finn E. Christensen ${ }^{8}$, William W. Craig ${ }^{6,9}$, Brian W. Grefenstette ${ }^{10}$, Jonathan E. Grindlay ${ }^{11}$, Fiona A. Harrison ${ }^{10}$, Jaesub Hong ${ }^{11}$, Kristin K. Madsen ${ }^{10}$, Melania Nynka ${ }^{1}$, Daniel Stern ${ }^{12}$, John A. Tomsick ${ }^{6}$, Daniel R. Wik ${ }^{13}$, Shuo Zhang ${ }^{1}$, William W. Zhang ${ }^{13}$ \& Andreas Zoglauer ${ }^{6}$

The Galactic Centre hosts a puzzling stellar population in its inner few parsecs, with a high abundance of surprisingly young, relatively massive stars bound within the deep potential well of the central supermassive black hole, Sagittarius A* (ref. 1). Previous studies suggest that the population of objects emitting soft $\mathrm{X}$-rays (less than 10 kiloelectronvolts) within the surrounding hundreds of parsecs, as well as the population responsible for unresolved $\mathrm{X}$-ray emission extending along the Galactic plane, is dominated by accreting white dwarf systems $s^{2-5}$. Observations of diffuse hard$\mathrm{X}$-ray (more than 10 kiloelectronvolts) emission in the inner 10 parsecs, however, have been hampered by the limited spatial resolution of previous instruments. Here we report the presence of a distinct hard-X-ray component within the central $4 \times 8$ parsecs, as revealed by subarcminute-resolution images in the 20-40 kiloelectronvolt range. This emission is more sharply peaked towards the Galactic Centre than is the surface brightness of the soft-X-ray population ${ }^{5}$. This could indicate a significantly more massive population of accreting white dwarfs, large populations of lowmass X-ray binaries or millisecond pulsars, or particle outflows interacting with the surrounding radiation field, dense molecular material or magnetic fields. However, all these interpretations pose significant challenges to our understanding of stellar evolution, binary formation, and cosmic-ray production in the Galactic Centre.

The Galactic Centre region is dense with X-ray-emitting objects ${ }^{6}$; it contains the supernova remnant Sagittarius (Sgr) A East, the colliding stellar winds surrounding Sgr A*, the hot plasma of the Sgr A East plume, dozens of magnetic X-ray filaments, and thousands of resolved ${ }^{7}$ and unresolved point sources that constitute the Galactic ridge X-ray emisison $^{3-5,8}$. In hard X-rays, the INTEGRAL satellite has detected emission centred within $1^{\prime}$ of the Galactic Centre ${ }^{9}$. However, the spatial resolution of INTEGRAL's IBIS coded aperture mask (12') has motivated speculation that the emission results not from a single object, but from a collection of the many surrounding X-ray sources ${ }^{3}$.

The NuSTAR X-ray observatory ${ }^{10}$, which has an effective area extending from 3 to $79 \mathrm{keV}$ and an angular resolution of $18^{\prime \prime}$ (equivalent to $0.7 \mathrm{pc}$ at the Galactic Centre), viewed the Galactic Centre for a total of $281 \mathrm{ks}$ in July, August and October of 2012. The image of the central $12 \mathrm{pc} \times 12 \mathrm{pc}$ of the Galaxy (Fig. 1) in the $20-40 \mathrm{keV}$ energy band reveals for the first time a faint diffuse emission that is peaked at the Galactic Centre and extends along the Galactic plane. The image is dominated by this feature, whose spectrum and localization within several parsecs of Sgr A* distinguish it from other unresolved X-ray emission in the Galaxy, and which has no obvious correlation with radio images of the dense molecular gas of the circumnuclear disk ${ }^{11}$ or the dust and gas of Sgr A West ${ }^{12}$. The features prominent in soft-X-ray images no longer visibly contribute, with the exception of bright pointlike emission from the pulsar wind nebula G359.95-0.04 ${ }^{13}$ and fainter emission from the X-ray filament G359.97-0.038 ${ }^{14}$ and the Cannonball ${ }^{15}$ neutron star.

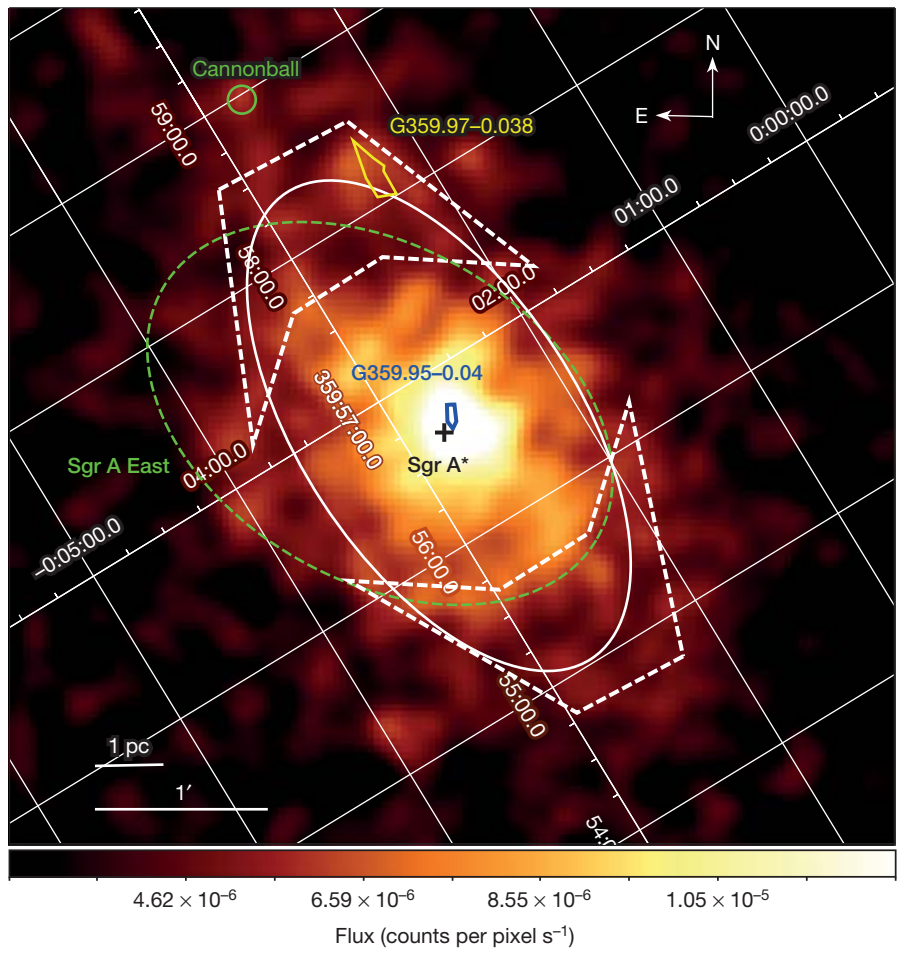

Figure $1 \mid$ The $20-40 \mathrm{keV}$ image of the inner $5^{\prime} \times 5^{\prime}(12 \mathrm{pc} \times 12 \mathrm{pc})$ of the Galaxy. The colour scale shows flux in units of counts per pixel $\mathrm{s}^{-1}$. The image has been smoothed with a Gaussian kernel of width $\sim 5^{\prime \prime}$ ( 2 pixels). The solid ellipse (white) illustrates the FWHM of the fit to the unresolved emission. Emission consistent with the pulsar wind nebula G359.95-0.04 ${ }^{13}$, as well as fainter emission from the Cannonball ${ }^{19}$ and non-thermal filament G359.97$0.038^{14}$, is also visible. The dashed ellipse (green) indicates the soft X-ray extent of Sgr A East ${ }^{19}$. Spectra are extracted from the two thick-dashed regions (white polygons).

${ }^{1}$ Columbia Astrophysics Laboratory, Columbia University, 550 West 120th Street, Room 1027, New York, New York 10027, USA. ${ }^{2}$ Haverford College, 370 Lancaster Avenue, KINSC L109, Haverford, Pennsylvania 19041, USA. ${ }^{3}$ Instituto de Astrofísica, Facultad de Física, Pontificia Universidad Católica de Chile, 306, Santiago 22, Chile. ${ }^{4}$ Millennium Institute of Astrophysics, Vicuña Mackenna 4860,

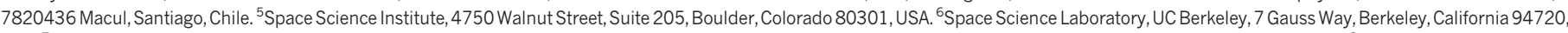
USA. ${ }^{7}$ Kavli Institute for Astrophysics and Space Research, Massachusetts Institute of Technology, 77 Massachusetts Avenue, 37-555, Cambridge, Massachusetts 02139, USA. ${ }^{8}$ DTU Space, National Space

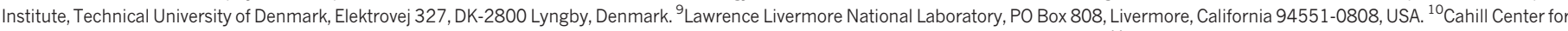

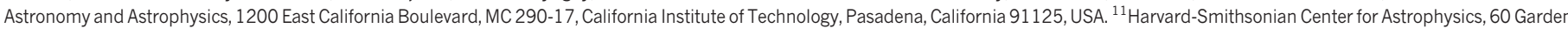
Street, MS-83, Cambridge, Massachusetts 02138, USA. ${ }^{12}$ Jet Propulsion Laboratory, California Institute of Technology, Pasadena, 4800 Oak Grove Drive, MS 169-221, California 91109, USA. ${ }^{13}$ NASA Goddard Space Flight Center, 8800 Greenbelt Road, Code 662, Greenbelt, Maryland 20771, USA. 

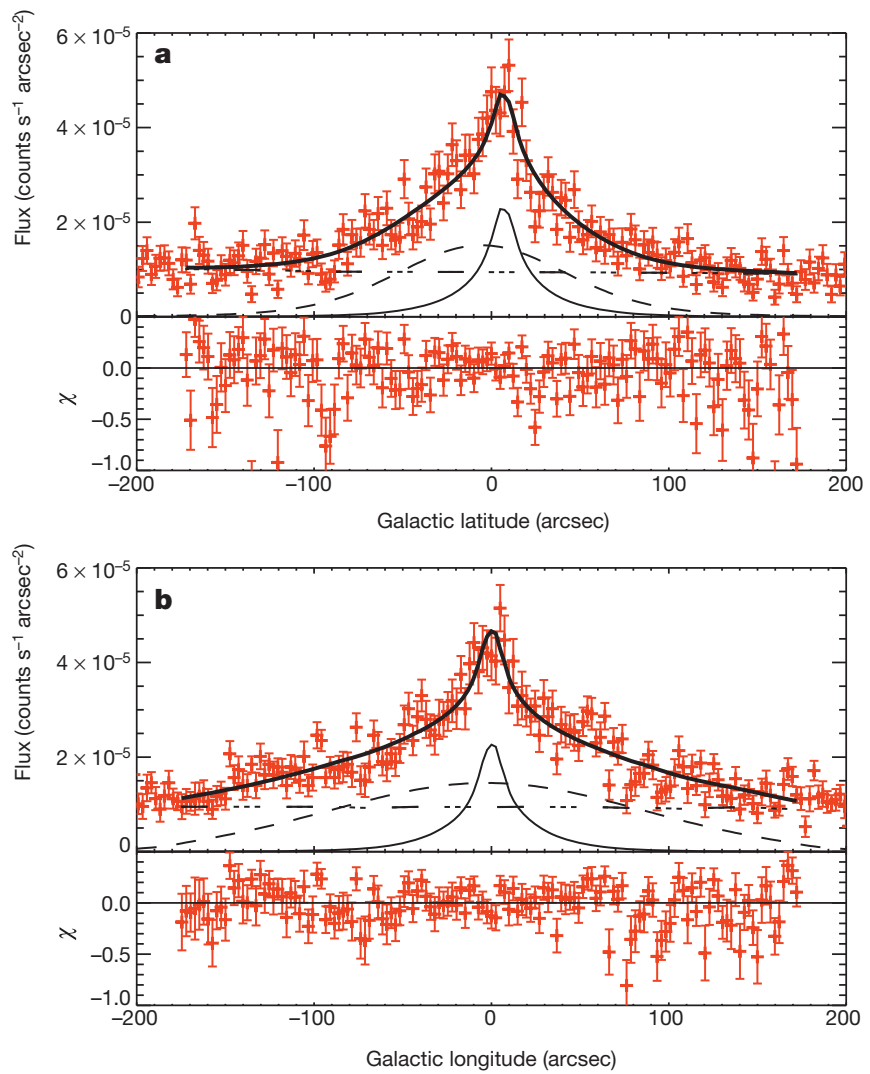

Figure $2 \mid$ Profiles of the $20-40 \mathrm{keV}$ data and spatial model along Galactic longitude and latitude. a, Galactic latitude; $\mathbf{b}$, Galactic longitude. Top panels, the data (flux in units of counts s${ }^{-1} \operatorname{arcsec}^{-2}$, red data points; error bars, $1 \sigma$ ) are fitted to a model consisting of an ellipsoidal Gaussian (dashed line), which describes the extended emission, and a point-like Gaussian (thin solid line), which describes G359.95-0.04, both convolved with the on-axis NuSTAR PSF, as well as a background (dash-dot line) that varies between detector chips. Profiles are integrated from $25^{\prime \prime}$ on either side of each axis, with the origin defined at Sagittarius A*. The combined model (thick solid line) and residual emission (lower panel) show that this model describes the data well.
The spatial distribution of this new feature can be described by an ellipsoidal Gaussian with a full-width at half-maximum (FWHM) of $7.8_{-0.7}^{+0.9} \mathrm{pc}$ along the Galactic plane and $3.9_{-0.3}^{+0.4} \mathrm{pc}$ out of the plane (Fig. 2), where the distance to the Galactic Centre is set to be $8 \mathrm{kpc}$, the physical extent is quoted before convolution with the NuSTAR point spread function (PSF), and errors are $3 \sigma$ confidence limits. The centroid of this emission is compatible with the Galactic Centre, defined as the radio location of Sgr A* (right ascension 17 h 45 min $40.036 \mathrm{~s}$, declination $-29^{\circ} 00^{\prime} 28.17^{\prime \prime}$; J2000.0 $)^{16}$, at the $2 \sigma$ confidence level. As the emission is centred $\sim 1^{\prime}$ away from the centre of the Sgr A East thermal emission and extends asymmetrically $>30^{\prime \prime}$ beyond the supernova shell to the southwest, a hard-X-ray component of Sgr A East is ruled out as the origin.

We performed spectral analysis in two regions at radii $r \approx 1^{\prime}-2^{\prime}$ to the southwest and northeast of Sgr A* (see Fig. 1), chosen such that $<4 \%$ of the emission from G359.95- 0.04 contaminates each region (radii are measured from the radio location of Sgr $A^{*}$ ). Since the NuSTAR spectral resolution ${ }^{10}$ is insufficient to fully resolve the neutral, He-like, and $\mathrm{H}$-like Fe emission lines, we used 2-10 keV XMMNewton data from these same regions to constrain the temperatures of the plasma emission below $20 \mathrm{keV}$.

The soft spectrum of the southwest region is dominated by point sources and truly diffuse emission (Fig. 3). Modelling this as two collisional-ionized plasmas ${ }^{17}$ yields the best-fit temperatures $k T_{1}=1.0_{-0.4}^{+0.3} \mathrm{keV}$ and $k T_{2}=7.5_{-1.3}^{+1.6} \mathrm{keV}$, with a neutral Fe equivalent width of $126_{-44}^{+46} \mathrm{eV}$ at $6.4 \mathrm{keV}$ (90\% confidence limits). These values are consistent with those previously obtained by Chandra analysis of faint point sources ${ }^{2}$ in the inner $9^{\prime}$ and diffuse emission ${ }^{18}$ in the inner $r \approx 2^{\prime}-17^{\prime}$, as well as XMM-Newton analysis ${ }^{5}$ of the regions $r=2^{\prime}-20^{\prime}$. The $k T \approx 1.0 \mathrm{keV}$ plasma is attributed to supernova heating of the interstellar medium, coronally active stars, and accreting non-magnetic white dwarfs ${ }^{4}$. The $k T \approx 7.5 \mathrm{keV}$ plasma is consistent with emission from resolved and unresolved accreting magnetic white dwarfs ${ }^{2,3,5}$ with mean white dwarf mass $M_{\mathrm{WD}} \approx 0.5 M_{\odot}\left(M_{\odot}\right.$ is the solar mass $)$. The stellar-mass $(M)$ normalized luminosity $(L)$ of our thermal components is $L(2-10 \mathrm{keV}) / M \approx 9 \times 10^{27} \mathrm{erg} \mathrm{s}^{-1} M_{\odot}^{-1}$ at $r \approx 3 \mathrm{pc}$, which is consistent with that measured by XMM-Newton ${ }^{5}$ at $r \approx 4 \mathrm{pc}$. We therefore conclude that our $k T_{1}$ and $k T_{2}$ components describe the same emission established by previous X-ray observations.

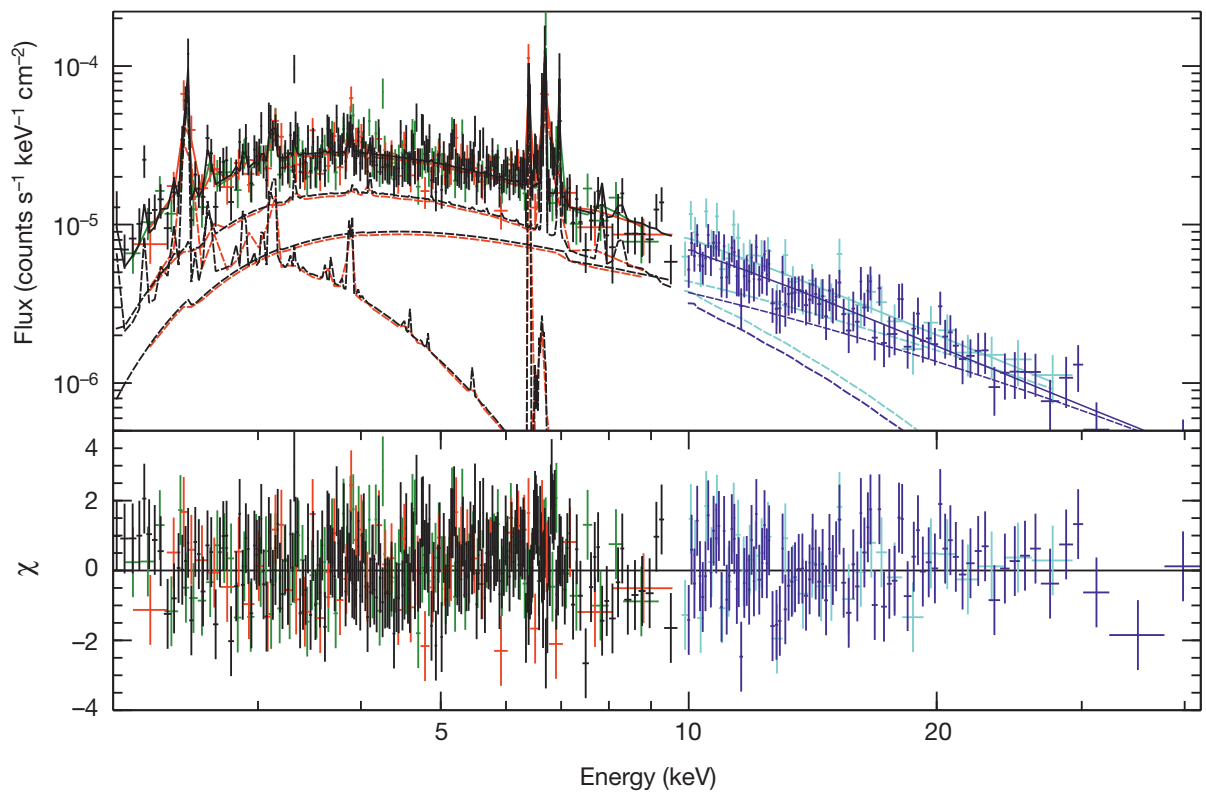

Figure 3 Unfolded broadband X-ray spectrum of the southwest region. Top panel, the 2-10 keV spectrum consists of XMM-Newton data from the pn (black), MOS1 (red) and MOS2 (green) instruments. Plotted on the $y$ axis is flux in units of counts s${ }^{-1} \mathrm{keV}^{-1} \mathrm{~cm}^{-2}$. The $10-40 \mathrm{keV}$ spectrum consists of NuSTAR focal plane A (dark blue) and B (cyan) data. Error bars are $1 \sigma$. Above
$20 \mathrm{keV}$, the extended emission dominates, described well by a $k T>35 \mathrm{keV}$ bremsstrahlung (here) or a photon index of $\Gamma=1.3-1.8$ (Extended Data Fig. 4). Dashed lines illustrate separate model components. Bottom panel, fit residuals. Full spectral parameters are given in Extended Data Tables 2 and 3. 
Above $20 \mathrm{keV}$, this thermal emission becomes negligible, and the spectrum is dominated by hard emission from our new feature. This emission can be described by either a power law with photon index $\Gamma=1.5_{-0.2}^{+0.3}$ and flux $F_{\text {power-law }}(20-40 \mathrm{keV})=6.7 \times 10^{-13} \mathrm{erg} \mathrm{s}^{-1}$ $\mathrm{cm}^{-2}$ or a poorly constrained high-temperature bremsstrahlung at $k T_{\text {high }}=58_{-23}^{+127} \mathrm{keV}$ and flux $F_{k T \text {-high }}(20-40 \mathrm{keV})=6.8 \times 10^{-13}$ erg $\mathrm{s}^{-1} \mathrm{~cm}^{-2}$. It is critical to note that this hard spectrum cannot be accounted for by extrapolating the previously detected soft thermal emission attributed to accreting magnetic white dwarfs.

The X-ray energy $E_{\mathrm{X}}>20 \mathrm{keV}$ spectrum and flux of the northeast region are consistent with those observed in the southwest. The northeast contains significant soft emission from Sgr A East, which dominates over the point source and diffuse populations. Sgr A East is modelled as a two-temperature collisional-ionized plasma, yielding temperatures $k T_{1}=1.1_{-0.2}^{+0.1} \mathrm{keV}$ and $k T_{2}=5.1_{-0.7}^{+0.9} \mathrm{keV}$, and metallicity $Z=2.3_{-0.4}^{+0.9} Z_{\odot}$ (where $Z_{\odot}$ is the solar metallicity), which are in agreement with Chandra and XMM-Newton measurements ${ }^{19,20}$. Again, this soft thermal emission does not contribute significantly above $20 \mathrm{keV}$, where the spectrum is fitted well by either a power law with $\Gamma=1.6_{-0.4}^{+0.3}$ and flux $F_{\text {power-law }}(20-40 \mathrm{keV})=7.6 \times 10^{-13}$ erg $\mathrm{s}^{-1} \mathrm{~cm}^{-2}$ or a high-temperature bremsstrahlung with $k T_{\text {high }}=66_{-30}^{+203} \mathrm{keV}$ and flux $F_{k T \text {-high }}(20-40 \mathrm{keV})=7.4 \times 10^{-13} \mathrm{erg}$ $\mathrm{s}^{-1} \mathrm{~cm}^{-2}$. Faint contaminating emission from the non-thermal X-ray filament G359.97-0.038 ${ }^{14}$ accounts for the 14\% difference in flux between the northeast and southwest regions.

The uniform 20-40 keV spectral and flux values in both regions support the conclusion that the feature is symmetric along the Galactic plane around Sgr A* and is either thermal with $k T_{\text {high }}>35 \mathrm{keV}$ or non-thermal with a photon index of $\Gamma=1.2-1.9$ (90\% confidence limits). Using the two-dimensional Gaussian spatial intensity model to scale the flux from the southwest region indicates a luminosity of $L(20-40 \mathrm{keV})=2.4 \times 10^{34} \mathrm{erg} \mathrm{s}^{-1}$ within the $4 \mathrm{pc} \times 8 \mathrm{pc}$ FWHM of emission. This is $<10 \%$ of the magnitude of the $20-40 \mathrm{keV}$ luminosity of the INTEGRAL Galactic Centre source.

The measured spectrum, luminosity and spatial profile constrain the possible origins of this emission, even given the broad parameter range of our phenomenological model. Truly diffuse sources, such as synchrotron radiation from magnetic filaments ${ }^{21}$ or low-surfacebrightness pulsar wind nebulae ${ }^{22}$, or bremsstrahlung and inverse Compton emission from Sgr A* particle outflows (cosmic rays) interacting with dense molecular material, could produce the observed spectrum. This interpretation is challenging, though, as there is no spatial correlation with corresponding radio images ${ }^{12}$ or radiation density models ${ }^{23}$, and our current estimates of magnetic filaments and supernova birth rates in this region cannot reproduce the observed luminosity (as described in Methods).

A natural explanation for our emission is provided by the intermediate polar (a type of cataclysmic variable binary star), which has the hardest spectrum of all accreting magnetic white dwarfs. A mean $k T>35 \mathrm{keV}$ implies a mean white dwarf mass $^{24} M_{\mathrm{WD}}>0.9 M_{\odot}$. This is substantially more massive than the population with mean $M_{\mathrm{WD}} \approx 0.5 M_{\odot}$ previously observed in the Galactic Centre ${ }^{2,5}$ and ridge ${ }^{3}$, or the $M_{\mathrm{WD}}=0.66_{-0.07}^{+0.09} M_{\odot}$ population observed in the Galactic bulge ${ }^{8}$. It is further distinguished from the low-mass intermediate polar population by its surface brightness distribution, which falls more steeply with distance from the Galactic Centre. Refitting our 20-40 keV image in the region $r>60$ " (to avoid biasing the distribution owing to the presence of G359.95-0.04) shows a decrease with angular offset from Sgr A*, $\theta$, and latitudinal angular offset from Sgr $\mathrm{A}^{*}, \phi$, of $\theta^{-\alpha} \exp \left(-|\phi| / \phi_{\text {sc }}\right)$, with $\alpha=1.4 \pm 0.1$ and latitudinal scale height $\phi_{\mathrm{sc}}=1.0^{\prime} \pm 0.1^{\prime}$ ( $1 \sigma$ errors). This is significantly narrower than the $\alpha=0.6_{-0.03}^{+0.02}$ and $\phi_{\mathrm{sc}}=18.6_{-1.2^{\prime}}^{+1.6^{\prime}}$ measured for the $2-10 \mathrm{keV}$ distribution $^{5}$ at $r>2^{\prime}$.

Our observed luminosity implies that about $10^{3}-10^{4}$ of these massive intermediate polars would exist in the central $4 \mathrm{pc} \times 8 \mathrm{pc}$. Scaling the density of intermediate polars in the solar neighbourhood by the stellar density of the Galactic Centre ${ }^{25}$ and the fraction of sufficiently massive progenitor B-stars predicts about three orders of magnitude fewer systems. Though puzzling, this overabundance is of similar magnitude to that implied for the low-mass population ${ }^{5}$.

A significant contribution from low-mass X-ray binaries or from millisecond pulsars may also be possible. The stellar density of faint $\mathrm{X}$-ray transients is already known to be higher in the Galactic Centre $^{26,27}$. However, for a population of low-mass black hole ${ }^{28}$ or neutron $\operatorname{star}^{29} \mathrm{X}$-ray binaries to account for the observed luminosity while remaining consistent with SWIFT X-ray outburst monitoring results $^{26}$, it must be composed of a rare class of very faint X-ray transients ${ }^{26,28}$. A sufficient population of millisecond pulsars would account for $\sim 40 \%$ of the sources identified by Chandra. This in contrast to previous analyses identifying accreting magnetic white dwarfs as the majority ${ }^{2,47}$, but could indicate a surprising abundance of unidentified millisecond pulsars in the region. This could strengthen the motivation for a millisecond pulsar explanation of the $\gamma$-ray excess observed by the Fermi $\gamma$-ray space telescope surrounding the Galactic Centre ${ }^{30}$.

Regardless of origin, these observations reveal novel processes present within the inner parsecs of the Galaxy. If arising from a population of compact objects, the steeply falling spatial distribution suggests that binary systems are accumulating, forming, and/or experiencing accretion with a much higher probability in the inner parsecs than in the solar neighbourhood or the Galactic bulge. These observations thus provide input useful for studying accretion physics, dynamical formation, and evolution of exotic binaries near the central supermassive black hole. A cosmic-ray origin, in contrast, has implications for models of particle outflow from Sgr $\mathrm{A}^{*}$, as well as radiation and magnetic field distributions.

Online Content Methods, along with any additional Extended Data display items and Source Data, are available in the online version of the paper; references unique to these sections appear only in the online paper.

\section{Received 15 December 2014; accepted 24 February 2015.}

1. Genzel, R., Eisenhauer, F. \& Gillessen, S. The Galactic Center massive black hole and nuclear star cluster. Rev. Mod. Phys. 82, 3121-3195 (2010).

2. Muno, M. P. et al. The spectra and variability of X-ray sources in a deep Chandra observation of the Galactic Center. Astrophys. J. 613, 1179-1201 (2004).

3. Krivonos, R. et al. Hard X-ray emission from the Galactic ridge. Astron. Astrophys. 463, 957-967 (2007).

4. Revnivtsev, M. et al. Discrete sources as the origin of the Galactic X-ray ridge emission. Nature 458, 1142-1144 (2009).

5. Heard, V.\& Warwick, R. XMM-Newton observations of the Galactic Centre region I: The distribution of low-luminosity X-ray sources. Mon. Not. R. Astron. Soc. 428, 3462-3477 (2013).

6. Goldwurm, A. in The Galactic Center: a Window to the Nuclear Environment of Disk Galaxies (eds Morris, M. R., Wang, Q. D. \& Yuan, F.) Sect. 3 394-401 (Astron. Soc. Pacif. Conf. Ser. Vol. 439, 2011)

7. Muno, M. P. et al. A catalog of X-ray point sources from two megaseconds of Chandra observations of the Galactic Center. Astrophys. J. Suppl. Ser. 181, 110-128 (2009)

8. Yuasa, T., Makishima, K. \& Nakazawa, K. Broadband spectral analysis of the Galactic ridge X-ray emission. Astrophys. J. 753, 129 (2012).

9. Bélanger, G. et al. A persistent high-energy flux from the heart of the Milky Way: INTEGRAL's view of the Galactic Center. Astrophys. J. 636, 275-289 (2006).

10. Harrison, F. A. et al. The Nuclear Spectroscopic Telescope Array (NuSTAR) highenergy X-ray mission. Astrophys. J. 770, 103 (2013).

11. Christopher, M. H., Scoville, N. Z., Stolovy, S. R. \& Yun, M. S. HCN and HCO observations of the Galactic circumnuclear disk. Astrophys. J. 622, 346-365 (2005)

12. Zhao, J.-H., Morris, M. R. \& Goss, W. M. in The Galactic Center: Feeding and Feedback in a Normal Galactic Nucleus (eds Sjouwerman, L. O., Lang, C. C. \& Ott, J.) 364-368 (IAU Symp. Vol. 303, 2014)

13. Wang, Q. D., Lu, F. J. \& Gotthelf, E. V. G359.95-0.04: an energetic pulsar candidate near Sgr A*. Mon. Not. R. Astron. Soc. 367, 937-944 (2006)

14. Nynka, M. etal. G359.97-0.038: a hard X-ray filament associated with a supernova shell-molecular cloud interaction. Astrophys. J. 800, 119 (2015).

15. Nynka, M. et al. High-energy X-rays from J174545.5-285829, the Cannonball: a candidate pulsar wind nebula associated with Sgr A East. Astrophys. J. 778, L31 (2013).

16. Menten, K. M., Reid, M. J., Eckart, A. \& Genzel, R. The position of Sagittarius A* accurate alignment of the radio and infrared reference frames at the Galactic Center. Astrophys. J. 475, L111-L114 (1997).

17. Smith, R., Brickhouse, N., Liedahl, D. \& Raymond, J. Collisional plasma models with APEC/APED: emission-line diagnostics of hydrogen-like and helium-like ions. Astrophys. J. 556, L91-L95 (2001). 
18. Muno, M. et al. Diffuse X-ray emission in a deep Chandra image of the Galactic Center. Astrophys. J. 613, 326-342 (2004).

19. Park, S. et al. A candidate neutron star associated with Galactic Center supernova remnant Sagittarius A East. Astrophys. J. 631, 964-975 (2005)

20. Sakano, M., Warwick, R. S., Decourchelle, A. \& Predehl, P. XMM-Newton observations of Sagittarius A East. Mon. Not. R. Astron. Soc. 350, 129-139 (2004).

21. Zhang, S. et al. High-energy X-ray detection of G359.89-0.08 (Sgr A-E): magnetic flux tube emission powered by cosmic rays? Astrophys. J. 784, 6 (2014).

22. Johnson, S. P., Dong, H. \& Wang, Q. D. A large scale survey of X-ray filaments in the Galactic Center. Mon. Not. R. Astron. Soc. 399, 1429-1440 (2009).

23. Davidson, J. et al. The luminosity of the Galactic Center. Astrophys. J. 387, 189-211 (1992).

24. Suleimanov, V., Revnivtsev, M. \& Ritter, H. RXTE broadband X-ray spectra of intermediate polars and white dwarf mass estimates. Astron. Astrophys. 435, 191-199 (2005).

25. Merrit, D. (ed.) Dynamics and Evolution of Galactic Nuclei Ch. 5276 (Princeton Series in Astrophysics, Princeton Univ. Press, 2013).

26. Degenaar, N. etal. A four-year XMM-Newton/Chandra monitoring campaign of the Galactic centre: analysing the X-ray transients. Astron. Astrophys. 545, A49-A73 (2012).

27. Muno, M. P. et al. An overabundance of transient X-ray binaries within $1 \mathrm{pc}$ of the Galactic Center. Astrophys. J. 622, L113-L116 (2005).

28. Menou, K., Narayan, R. \& Lasota, J.-P. A population of faint nontransient low-mass black hole binaries. Astrophys. J. 513, 811-826 (1999).

29. Heinke, C. et al. Discovery of a second transient low-mass $X$-ray binary in the globular cluster NGC 6440. Astrophys. J. 714, 894-903 (2010).

30. Hooper, D. \& Linden, T. On the origin of the gamma rays from the Galactic Center. Phys. Rev. D 84, 123005 (2011).
Acknowledgements This work was supported by NASA contract no. NNG08FD60C, and made use of data from the NuSTAR mission, a project led by the California Institute of Technology, managed by the Jet Propulsion Laboratory, and funded by NASA. We thank the NuSTAR Operations, Software and Calibration teams for support with the execution and analysis of these observations. This research has made use of the NuSTAR Data Analysis Software (NuSTARDAS) jointly developed by the ASI Science Data Center (ASDC, Italy) and the California Institute of Technology (USA). We also thank A. Canipe, J. Dodaro, D. Hong and T.V. T. Luu for assistance with data preparation and analysis. F.E.B. acknowledges support from Basal-CATA PFB-06/2007, CONICYT-Chile (FONDECYT 1141218 and EMBIGGEN Anillo ACT1101), and Project IC120009 "Millennium Institute of Astrophysics (MAS)" funded by the Iniciativa Científica Milenio del Ministerio de Economía, Fomento y Turismo.

Author Contributions K.P.: image analysis, spectral analysis, modelling of the Sagittarius A* region, interpretation and manuscript preparation. C.J.H.: interpretation, manuscript preparation and review. F.E.B.: interpretation and manuscript review. R.K. image analysis and interpretation. K.M.: interpretation, manuscript preparation and review. F.K.B., N.M.B., S.E.B., J.E.G., J.H. and J.A.T.: interpretation and manuscript review. F.E.C., W.W.Z.: optics production and calibration. W.W.C.: optics and instrumentation production and response, and observation planning. B.W.G., K.K.M., D.R.W. and A.Z.: software for data analysis, background modelling, and calibration. F.A.H.: NuSTAR principal investigator, observation planning, interpretation and manuscript review. M.N., S.Z.: data preparation and interpretation. D.S.: observation planning and manuscript review.

Author Information Reprints and permissions information is available at www.nature.com/reprints. The authors declare no competing financial interests. Readers are welcome to comment on the online version of the paper. Correspondence and requests for materials should be addressed to K.P. (kperez1@haverford.edu). 


\section{METHODS}

We use data from three NuSTAR observations of the Sgr A* region in 2012: 20-23 July (154 ks), 4-6 August ( $77 \mathrm{ks})$, and 16-18 October (50 ks). These correspond to the observation IDs 30001002001, 30001002003, and 30001002004, respectively. Data reduction and spectral extraction were performed with the NuSTAR Data Analysis Software (NuSTARDAS) v1.1.0 software package. The HEASoft v6.13 package was used for data analysis.

Event selection. We removed all data from within $100 \mathrm{~s}$ of passage through the South Atlantic Anomaly and time periods corresponding to Sgr A* flares, as observed by NuSTAR or during coincident Chandra observations ${ }^{31}$. Data from focal plane B of the July and October observations are not included due to stray light ${ }^{10}$ from the nearby bright objects GX $3+1$ and 1E1740-2942. After data screening and combining available focal plane A (FPMA) and B (FPMB) observations, the analysis contains $\sim 297 \mathrm{ks}$ of effective exposure time.

Image analysis. The nominal NuSTAR image coordinates are accurate to 8 " $(90 \%$ confidence level $)^{10}$. To improve our positional accuracy, we derived an astrometric correction for each observation by registering known sources. For the July and October observations, we used the radio position of Sgr A*16, the NuSTAR position of the Sgr A* flare in the 3-79 keV band, the Chandra position of Sgr A-E ${ }^{22}$, and the NuSTAR position of Sgr A-E ${ }^{21}$ in the $3-10 \mathrm{keV}$ band for source registration. For the August observation, where the lack of a flare makes localization of Sgr $A^{*}$ difficult with NuSTAR, we instead used the Chandra position of the Cannonball ${ }^{19}$, the NuSTAR position of the Cannonball ${ }^{15}$ in the $10-40 \mathrm{keV}$ band, the Chandra position of Sgr A-E, and the NuSTAR position of Sgr A-E in the 3-10 $\mathrm{keV}$ band.

After each photon event file was position corrected, we combined NuSTAR images in sky coordinates and normalized the resulting image by an effective exposure map that accounts for observation time, but not vignetting effects. Since the optical axis was near Sgr A* for all observations and detector background rates in the $20-40 \mathrm{keV}$ energy band are non-negligible, vignetting was accounted for in the spatial fitting by convolving the source components, but not detector background components, with the on-axis NuSTAR point spread function (PSF). We defined a systematic error on the astrometric correction to be the maximum distance between the reference positions and the NuSTAR centroid positions of the Cannonball and Sgr A-E knot in the resulting image, yielding uncertainties of $1.0^{\prime \prime}$ in RA and 1.2" in Dec. This is in addition to the 4 " spatial uncertainty from the PSF smearing effect due to incomplete aspect reconstruction ${ }^{32}$.

We used the Sherpa ${ }^{33}$ package to fit a phenomenological model to the 20-40 $\mathrm{keV}$ raw photon count sky image. Due to low source counts, the C-statistic was used. This model was composed of one symmetric point-like Gaussian and one ellipsoidal extended Gaussian source, both convolved with the on-axis NuSTAR PSF stored in the NuSTARDAS v1.1.0 CALDB database, and a flat internal background that varies between detector chips. The background map was derived using blank sky observations to fix the relative normalization of the detector background between each chip. In this energy range, the stray light background due to the cosmic X-ray background and Galactic ridge X-ray emission are subdominant compared to the internal background. The overall normalization of the background map was left as an independent fit parameter. The total effective exposure map was used to normalize all three fit components. The final model used for fitting was thus:

$$
\text { Psf } \times(\text { gauss } 2 \text { d.sourceOne }+ \text { gauss } 2 \text { d.sourceTwo }) * \text { emap }
$$

\section{+ bkgdmap $*$ emap}

where psf is the on-axis NuSTAR PSF, gauss2d.sourceOne is the point-like Gaussian, gauss2d.sourceTwo is the ellipsoidal extended Gaussian, bkgdmap is the background map, and emap is the effective exposure map. Here, $*$ indicates multiplication and $X$ indicates convolution. To minimize variations in background and detector response across different detector chips, and to avoid emission from the molecular cloud regions to the northeast, the fit was performed within a radius $r<3^{\prime}$ from the Galactic Centre.

The $20-40 \mathrm{keV}$ model and residuals in the fit region are shown in Extended Data Fig. 1. No remaining significant structure is present in the residual map. The component gauss2d.sourceOne fits to a FWHM of $1.8_{-0.7}^{+2.3^{\prime \prime}}$, where $2.5^{\prime \prime}$ is one image pixel. This is a lower limit on the spatial extent, consistent with a source small compared to the $18^{\prime \prime}$ (FWHM) NuSTAR PSF. The second component fits to a Gaussian with $\mathrm{FWHM}=195.8_{-16.8}^{+21.9^{\prime \prime}}$ along the major axis, $\mathrm{FWHM}=101.8_{-8.7}^{+11.4^{\prime \prime}}$ along the minor axis, and an inclination of $\theta=57^{\circ}$ from the positive northern axis. The centroid of this component is located at $\triangle \mathrm{RA}=-2.2_{-3.8}^{+4.5^{\prime \prime}}$ and $\Delta$ Dec. $=2.8_{-4.1}^{+3.4^{\prime \prime}}$ from Sgr A*. Because calculating fit errors while leaving all parameters free was too computationally intensive, the amplitude of the background map and the extended component ellipticity and $\theta$ were fixed during error calculation. All fit parameters are listed in Extended Data Table 1. Consistent values of centroid location and spatial extent are obtained by fitting a single ellipsoidal Gaussian and background model in a region that excludes the central $60^{\prime \prime}$ radius, thus minimizing emission from G359.95-0.04.

To compare the NuSTAR 20-40 keV morphology with the 2-10 keV surface brightness distribution, we re-fitted the NuSTAR data with the spatial model used by the Heard andWarwick analysis of XMM-Newton data in the region $r=2^{\prime}-20^{\prime}$ :

$$
S=N \theta^{-\alpha} \exp \left(-|\phi| / \phi_{\text {sc }}\right)+\text { const. }
$$

This model describes a surface brightness $S$ falling as a function of the angular offset $\theta$ (measured in arcminutes) from the position of Sgr $\mathrm{A}^{*}$ and the latitude angle $\phi$, measured with respect to the latitude of Sgr A*. The parameters $N, \alpha$ and $\phi_{\mathrm{sc}}$ represent the normalization of the power law, the power-law index, and the latitudinal scale height in arcminutes, respectively.

To avoid biasing the steepness of the distribution, we excluded the central $r<$ $60^{\prime \prime}$ region that contains the bright pulsar wind nebula G359.95-0.04. We also exclude the emission from $r<20^{\prime \prime}$ surrounding the Cannonball ${ }^{15}$. The constant background term const. is obtained by fitting the same background map as used in our two-Gaussian phenomenological model to the region $r=300^{\prime \prime}-400^{\prime \prime}$, yielding the same background normalization as obtained in the two-Gaussian model above. We note that this const. term does not represent the true underlying sky surface brightness, as it contains a large contribution from instrumental background. Fitting in the region $r=60^{\prime \prime}-180^{\prime \prime}$ constrains the spatial parameters to be $\alpha$ $=1.4 \pm 0.1$ and $\phi_{\mathrm{sc}}=1.0^{\prime} \pm 0.1^{\prime}$ ( $1 \sigma$ errors). This is significantly narrower in both longitude and latitude than the soft distribution observed by Heard and Warwick, who obtained $\alpha=0.6_{-0.03}^{+0.02}$ and $\phi_{\mathrm{sc}}=18.6_{-1.2}^{+1.6^{\prime}}$. The effect of vignetting is not important this close to the optical axis. Re-fitting the data using an exposure map that includes the effect of vignetting, calculated at $25 \mathrm{keV}$, yields consistent values of $\alpha=1.2 \pm 0.1$ and $\phi_{\mathrm{sc}}=1.0^{\prime} \pm 0.1^{\prime}$.

In order to fit over the full $r<180^{\prime \prime}$ region and to further compare with our twoGaussian model, we fix the magnitude and position of the point-like Gaussian describing G359.95-0.04 to the same values as listed in Extended Data Table 1. Re-fitting the distribution $S$ in this full region yields $\alpha=1.0^{\prime} \pm 0.1^{\prime}$ and $\phi_{\mathrm{sc}}=1.1^{\prime}$ $\pm 0.1^{\prime}$. The profiles of the total $20-40 \mathrm{keV}$ emission along Galactic longitude and latitude, along with this best-fit model result, are shown in Extended Data Fig. 2. Spectral analysis. Using the event files with astrometric corrections applied, we extracted source spectra from the dashed regions shown in Fig. 1. Since the 20-40 $\mathrm{keV}$ NuSTAR image is dominated by internal detector background, the background spectra were extracted from regions located on the same detector chip that contains the Sgr A* region, but outside the molecular cloud regions (see Extended Data Fig. 3).

We used 2-10 keV data from the XMM-Newton observations carried out on 2012 August 31 (observation ID 0694640301) and 2012 September 24 (observation ID 0694641101), reprocessed with SAS v12.0.1. No bright X-ray outbursts occurred during these periods. We extracted EPIC-pn and EPIC-MOS spectra from the regions used for the NuSTAR analysis and followed the standard data screening procedures from the September 2013 XMM-Newton ABC guide.

The resulting data from the MOS1, MOS2, and pn instruments sum to $\sim 210 \mathrm{ks}$ of effective exposure time. For MOS1, the 2012 September 24 observation fell on a dead detector chip and is not used. Since the XMM-Newton spectra are dominated by soft diffuse emission that fills the central arcminutes, the background spectra were extracted from dimmer regions located at least $10^{\prime}$ to the northwest of the Sagittarius A* region.

Spectral fitting and flux derivations were performed in XSPEC version 12.8.0 with photoionization cross-sections as defined in ref. 35 and abundances for the interstellar absorption as defined in ref. 36. All spectra were re-binned so that each spectral bin contained at least 20 counts above the estimated background level, obtained by scaling the background spectrum to the source extraction area.

To analyse the southwest and northeast region spectra of XMM-Newton and NuSTAR, we compared the models const.*tbabs*(apec + apec + gaus + po) and const.*tbabs*(apec + apec + gaus + bremss), where const. is a constant factor, tbabs is the neutral hydrogen absorption model, apec is a collisionally ionized plasma model, gaus is a Gaussian emission line model centred at $6.4 \mathrm{keV}$ with width fixed to $\sigma=0.01 \mathrm{keV}$ (to model neutral Fe emission), po is a power-law model, and bremss is a thermal bremsstrahlung model. The final spectral fit values are not significantly sensitive to the width of the Gaussian fixed at $6.4 \mathrm{keV}$. The thermal bremsstrahlung model was chosen to describe possible very-high-temperature $(>30 \mathrm{keV})$ emission, where emission lines would no longer be present. To account for different normalizations between instruments, the constant factor was allowed to fit freely to each data set.

The best-fit parameters and $90 \%$ confidence level statistical errors are listed in Extended Data Tables 2 and 3. In the southwest region, the lower-temperature component has a best-fit metallicity that is very high, $Z=5 Z_{\odot}$, but varying this parameter has no effect on the spectral parameters of the higher-energy compo- 
nents. The higher-temperature component fits to a metallicity $Z=1.7 Z_{\odot}$. This is consistent with emission from unresolved magnetic accreting white dwarfs, with slightly enhanced metallicity because the lighter elemental abundances, which are measured to be $Z>Z_{\odot}$ in the Galactic Centre ${ }^{18}$, are linked to the Fe abundance in the apec model. Because a degeneracy exists between the values for the abundance of the $k T_{2}$ component, the normalization of the $k T_{2}$ component, and the normalization of the power-law component, the $k T_{2}$ abundance was fixed to its best-fit value during the calculation of parameter error intervals. The hard component can be modelled equally well by either a thermal bremsstrahlung, as shown in Fig. 3, or a power law, as shown in Extended Data Fig. 4. In the northeast region, the hard emission can be described by these same models, as shown in Extended Data Figs 5 and 6. The non-thermal spectrum of G359.97- $0.038^{14}$ could contribute at most $19 \%$ of the flux observed in the northeast region (at the $90 \%$ confidence level). Spectral analysis of central $r=20^{\prime \prime}$. Our spatial model and the asymmetric surface brightness distribution indicate that our observation cannot be attributed to PSF leakage from a bright, central source. To further support this claim, we perform spectral analysis of the $r=20$ " region surrounding the point-like source attributed to G359.95-0.04. The radius was chosen to maximize the ratio of the point-source flux to the underlying extended flux. The software versions, background regions, and spectral re-binning are identical to those used above.

We first fit the NuSTAR data in the energy range $20-60 \mathrm{keV}$ with a power-law model, po, yielding $\Gamma=2.2_{-0.3}^{+0.4}$. This is significantly softer than the emission measured in the southwest or northeast regions. To better constraint the photon index, we again combine the 2-10 keV XMM-Newton data with $10-60 \mathrm{keV}$ NuSTAR data. The soft emission is dominated by Sgr A East, with best-fit temperatures and abundances consistent with Chandra and XMM-Newton measurements of this region ${ }^{19,20}$. The hard emission is described by $\Gamma=2.0_{-0.2}^{+0.2}$, again significantly softer than the best-fit values of the extended emission.

Population analysis. Truly diffuse sources. Chandra has identified $17 \mathrm{X}$-ray-emitting non-thermal filaments in the central $20^{\prime} \times 20^{\prime}$ region $^{22}$, with photon indices ranging within $\Gamma \approx 1.0-2.0$. Extrapolating the combined $2-10 \mathrm{keV}$ luminosity of the six filaments (excluding G359.95-0.04) that lie within the central $4 \mathrm{pc} \times 8 \mathrm{pc}$ region using our best-fit $\Gamma=1.6$, we see that they could contribute at most $30 \%$ of the observed luminosity. Also, no X-ray filaments are identified in our southwest region. The faintest filament observed by Chandra has a luminosity of $L(2-10 \mathrm{keV})$ $=2.0 \times 10^{32} \mathrm{erg} \mathrm{s}^{-1}$. Interpreting this as a minimum Chandra detection threshold and again extrapolating using the best-fit photon index implies that $\sim 100$ additional filaments would be necessary to account for the total luminosity. Radio emission, which is observed from $\sim 25 \%$ of X-ray filaments ${ }^{37}$, would thus be expected to trace our observed morphology. However, no such similar structure is observed in recent $6 \mathrm{~cm}$ radio maps ${ }^{12}$.

A population of low-surface-brightness pulsar wind nebulae $e^{38}$ could also contribute. Again assuming $L(2-10 \mathrm{keV})=2.0 \times 10^{32} \mathrm{erg} \mathrm{s}^{-1}$ is the minimum luminosity at which Chandra would detect an extended object, $\sim 100$ such objects are required. However, simulations indicate that only a few dozen should exist, spread over the central $r=20^{\prime}$ of the Galaxy ${ }^{22}$. Thus an order of magnitude higher supernova birth rate in the Galactic Centre than currently assumed would be required to explain our observations.

In addition to particle outflows from Sgr $\mathrm{A}^{*}$, cosmic rays from shocked stellar winds combined with the high radiation density of the region could produce an inverse Compton origin. To account for the observed 20-40 keV luminosity of the southwest region, which is at a distance of $r \approx 2-5 \mathrm{pc}$, a cosmic-ray density similar to that of the central $r<1 \mathrm{pc}$ and a radiation density of $\sim 10,000 \mathrm{eV} \mathrm{cm}^{-3}$ would be required $^{39}$. However, the radiation density decreases rapidly with distance from Sgr $\mathrm{A}^{* 23}$, falling from $\sim 5,000 \mathrm{eV} \mathrm{cm}^{-3}$ in the inner parsec to $\sim 50 \mathrm{eV} \mathrm{cm}^{-3}$ at a distance of $10 \mathrm{pc}$, and the cosmic-ray density is also expected to decrease rapidly away from the Galactic Centre.

A more exotic origin of electrons could be the annihilation of dark-matter particles. However, the electron energies required to produce the observed spectrum are too large to be produced by even the heaviest dark-matter candidates ${ }^{40}$. The NuSTAR emission is also much more centrally localized and asymmetric than would be predicted for dark-matter annihilation in the Galactic halo ${ }^{41}$.

Intermediate polars. Our estimate of the necessary number of intermediate polars to reproduce the observed spectrum is based on assumed ${ }^{8} 2-10 \mathrm{keV}$ luminosities ranging from $L_{\min } \approx 10^{30}-10^{31} \mathrm{erg} \mathrm{s}^{-1}$ to $L_{\max } \approx 10^{33} \mathrm{erg} \mathrm{s}^{-1}$, with an integral source luminosity distribution $N(>L) \propto L^{-\alpha}$ and spectral index $\alpha \approx 1-1.5$.

The bremsstrahlung model we have used in our spectral fitting approximates the spatially dependent temperature of the cooling accretion column of an intermediate polar by an average colour temperature. To directly determine the mean mass limit implied by our observations, we refit the spectra of the southwest and northeast regions using the model of ref. 24. Since our soft components agree with previously measured values, we constrain these parameters to stay within their $90 \%$ confidence intervals. This yields a $90 \%$ confidence level lower limit on the mean white dwarf mass of $M_{\mathrm{WD}}>0.9 M_{\odot}$. As with the high-temperature bremsstrahlung model, the upper limit is not well constrained.

Low-mass X-ray binaries. Our knowledge of the luminosity of quiescent black hole low-mass X-ray binaries is limited, based on a sample of only 17 systems ${ }^{42}$. For typical luminosities of $L(2-10 \mathrm{keV}) \approx(2-4) \times 10^{31} \mathrm{erg} \mathrm{s}^{-1}, \sim 10^{3}$ such systems would be required inside the central $4 \mathrm{pc} \times 8 \mathrm{pc}$ to account for our observed luminosity. In the last decade, $\mathrm{X}$-ray monitoring surveys ${ }^{26,43}$ are believed to have uncovered virtually all transient systems within the inner $50 \mathrm{pc}$ with recurrence times of less than $\sim 5-10 \mathrm{yr}$, outburst durations longer than a few days, and outburst luminosities $L(2-10 \mathrm{keV})>\sim 10^{34} \mathrm{erg} \mathrm{s}^{-1}$. Assuming a black hole binary outburst recurrence time of $T_{\mathrm{r}} \approx 50-100 \mathrm{yr}$ and durations ranging from weeks to months $s^{28}$ and using the integrated Swift monitoring time implies $\sim 12-50$ transient events should have been detected from an underlying population of $\sim 10^{3}$ black hole systems. However, only two new transient sources that could plausibly be attributed to low-mass black hole systems have been observed in that time. A large population of neutron star low-mass X-ray binaries is also inconsistent with $\mathrm{X}$-ray monitoring surveys, assuming a mean $L(2-10 \mathrm{keV}) \approx 10^{32} \mathrm{erg} \mathrm{s}^{-1}$ and recurrence times ${ }^{29}$ of $\sim 5-10 \mathrm{yr}$.

Millisecond pulsars. Some millisecond pulsars exhibit non-thermal emission with a typical photon index $\Gamma \approx 1-2$ (ref. 44), consistent with our observed spectrum. To study the X-ray luminosities of these objects, we selected 125 millisecond pulsars from the Australia Telescope National Facility Pulsar Catalog ${ }^{45}$ with spin periods and dipole magnetic field strengths consistent with the observed hard-X-ray emitting millisecond pulsars, $P<10 \mathrm{~ms}$ and $B<10^{11} \mathrm{G}$. The spin-down power of the selected objects, $\dot{E}$, ranges from $4 \times 10^{32}$ to $2 \times 10^{36} \mathrm{erg} \mathrm{s}^{-1}$, with a mean $\dot{E}=6 \times$ $10^{34} \mathrm{erg} \mathrm{s}^{-1}$. Using the relation between non-thermal luminosity and spin-down power established by previous studies ${ }^{46}, L(2-10 \mathrm{keV}) \approx 10^{-4 \dot{E}}$, we obtain a mean non-thermal X-ray luminosity of $L(2-10 \mathrm{keV})=6 \times 10^{30} \mathrm{erg} \mathrm{s}^{-1}$. Approximately 3,000 millisecond pulsars with this mean X-ray luminosity would be required to account for the our observations. Over 100 of these sources would have a luminosity of $L(2-10 \mathrm{keV})>\sim 4 \times 10^{31} \mathrm{erg} \mathrm{s}^{-1}$, which is the Chandra point-source detection limit for this region ${ }^{7}$. This implies that over $40 \%$ of the sources identified by Chandra within the central $4 \mathrm{pc} \times 8 \mathrm{pc}$ are millisecond pulsars, a claim that requires further study and is inconsistent with the intermediate polar interpretation of the majority of these sources ${ }^{2,4,7}$.

Sample size. No statistical methods were used to predetermine sample size.

31. Barrière, N. M. et al. NuSTAR detection of high-energy X-ray emission and rapid variability from Sagittarius A* flares. Astrophys. J. 786, 46 (2014).

32. An, H. et al. In-flight PSF calibration of the NuSTAR hard X-ray optics. Proc. SPIE 9144, 1-10 (2014).

33. Fruscione, A. A. et al. CIAO: Chandra's data analysis system. Proc. SPIE 6270, 1-12 (2006).

34. Arnaud, K. A. in Astronomical Data Analysis Software and Systems V (eds Jacoby, J. H \& Barnes, J.) 17-20 (ASP Conf. Ser. Vol. 101, 1996).

35. Verner, D. A., Ferland, G. J., Korista, K. T. \& Yakovlev, D. G. Atomic data for astrophysics II. New analytic FITS for photoionization cross sections of atoms and ions. Astrophys. J. 465, 487-510 (1996).

36. Wilms, J., Allen, A. \& McCray, R. On the absorption of X-rays in the interstellar medium. Astrophys. J. 542, 914-924 (2000).

37. Lu, F. J., Yuan, T. T. \& Lou, Y.-Q. An imaging and spectral study of 10 X-ray filaments around the Galactic Center. Astrophys. J. 673, 915-927 (2008).

38. Bamba, A. et al. X-ray evolution of pulsar wind nebulae. Astrophys. J. 719, L116 (2010).

39. Quataert, E. \& Loeb, A. Nonthermal THz to TeV emission from stellar wind shocks in the Galactic Center. Astrophys. J. 635, L45-L48 (2005).

40. Cirelli, M., Kadastik, M., Raidal, M. \& Strumia, A. Model-independent implications of the $\mathrm{e}^{ \pm}, \bar{p}$ cosmic ray spectra on properties of Dark Matter. Nucl. Phys. B 813, 1-21 (2009).

41. Fan, J., Katz, A., Randall, L. \& Reece, M. Double-disk dark matter. Phys. Dark Univ. 2, 139-156 (2013).

42. Homan, J. etal. The X-ray properties of the black hole transient MAXI J1659-152 in quiescence. Astrophys. J. 775, 9 (2013).

43. Degenaar, N. \& Wijnands, R. A four-year baseline Swift study of enigmatic X-ray transients located near the Galactic Center. Astron. Astrophys. 524, A69 (2010).

44. Zavlin, V. E. XMM-Newton observations of four millisecond pulsars. Astrophys. J. 638, 951-962 (2006).

45. Manchester, R. N., Hobbs, G. B., Teoh, A. \& Hobbs, M. The Australia Telescope National Facility pulsar catalogue. Astron. J. 129, 1993-2006 (2005).

46. Takata, J., Cheng, K. S. \& Taam, R. E. X-ray and gamma-ray emissions from rotation powered millisecond pulsars. Astrophys. J. 745, 100 (2012). 


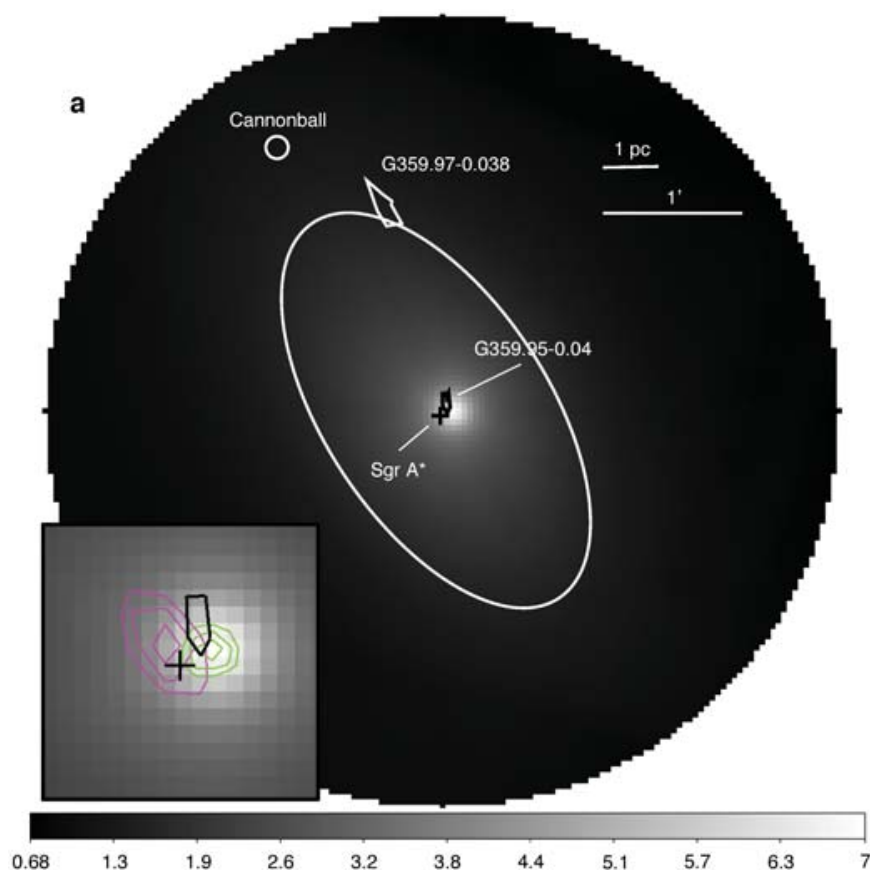

Extended Data Figure $1 \mid$ The best-fit $20-40 \mathrm{keV}$ spatial model and residual image. a, The model, consisting of a point-like Gaussian, an extended Gaussian, and a background model as described in the main text. The radio position of Sgr $\mathrm{A}^{* 16}$ and the Chandra positions of the Cannonball ${ }^{19}$, G359.95-0.04 $4^{13}$ and G359.97-0.038 $8^{22}$ are overlaid. The FWHM extent of the two-dimensional Gaussian fit to the extended emission is indicated by the solid ellipse. The image is shown with a linear grey-scale distribution with the range

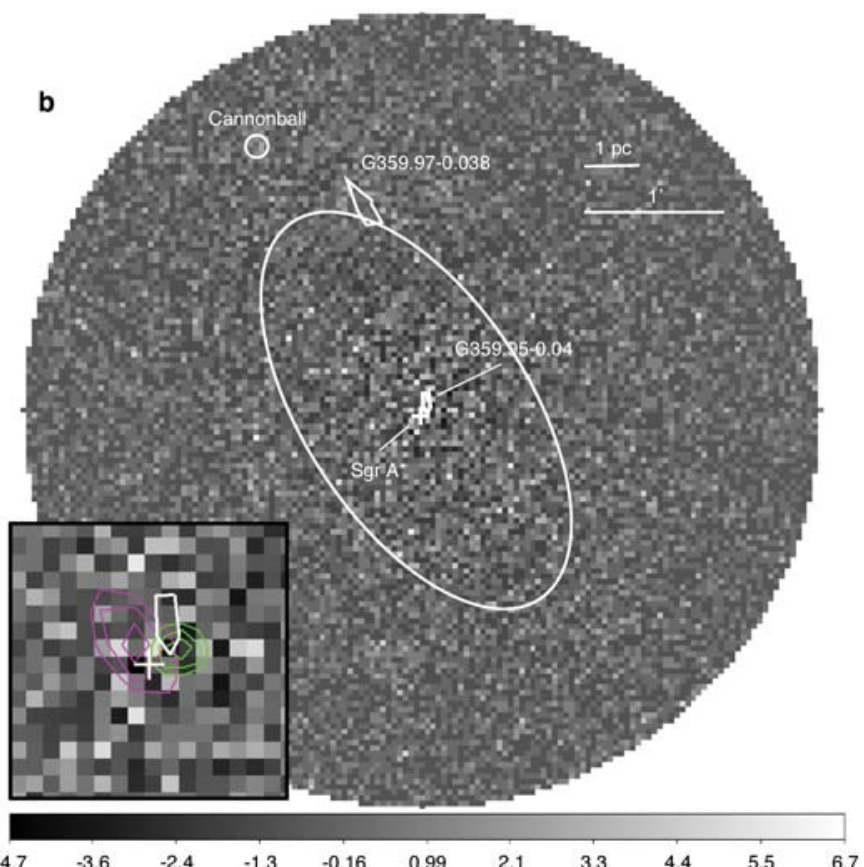

chosen to highlight the extended emission, in units of total counts per pixel (bar at bottom). b. The residual image (observed minus model). Insets in $\mathbf{a}$ and b show a magnified view of the central $40^{\prime \prime} \times 40^{\prime \prime}$ of the main panels, with $1 \sigma, 2 \sigma$ and $3 \sigma$ error contours on the centroid positions of the extended (magenta) and point-source (green) two-dimensional Gaussian models, as well as the position of Sgr A* (cross) and G359.95-0.04 (polygon). 

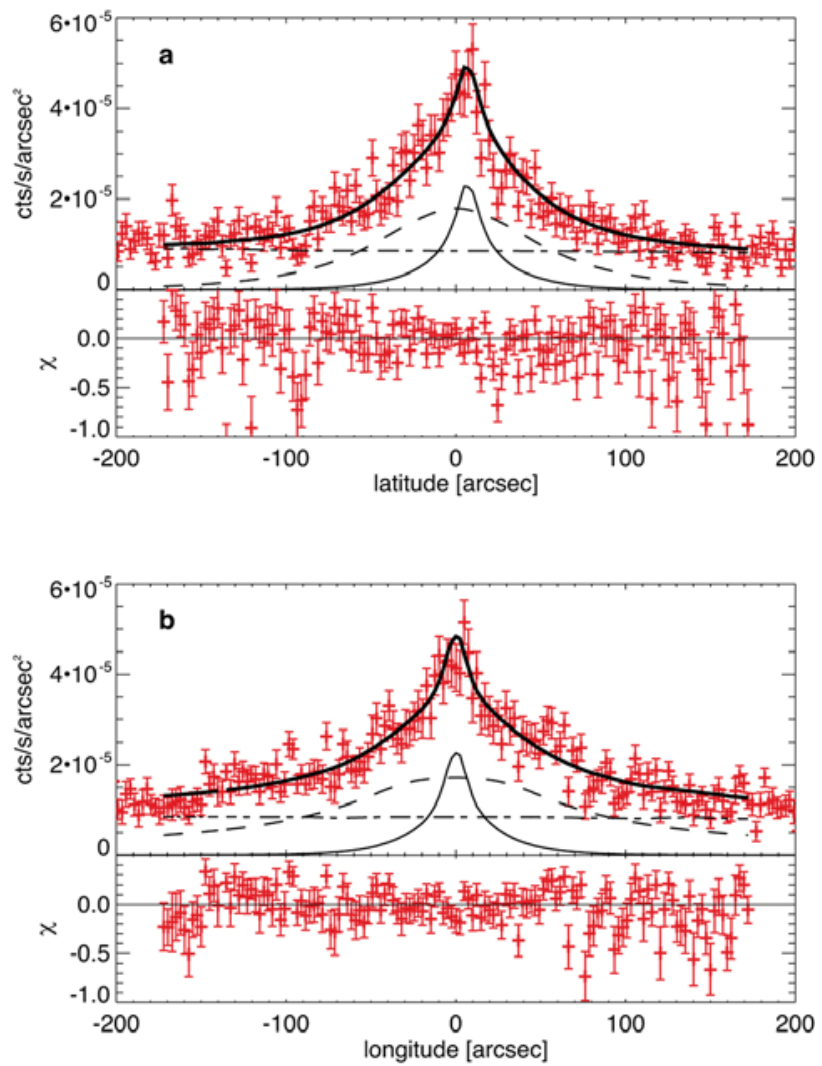

Extended Data Figure $2 \mid$ Profiles of the $20-40 \mathrm{keV}$ data and exponential spatial model along Galactic longitude and latitude. a, Galactic latitude; b, Galactic longitude. Top panels, the data (red, $1 \sigma$ errors) are fitted to a twodimensional model consisting of the surface brightness model used in ref. 5 and described in the main text (dashed line) and a point-like Gaussian source (thin solid line) that describes the emission from G359.95-0.04, both convolved with the on-axis NuSTAR PSF, as well as a flat background (dash-dot line) that varies between detector chips. Profiles are obtained by integrating from $25^{\prime \prime}$ on either side of each axis, with the origin defined at the position of Sgr A*. The combined model (thick solid line) and residual emission (lower panels) show that this phenomenological model describes the data well. 


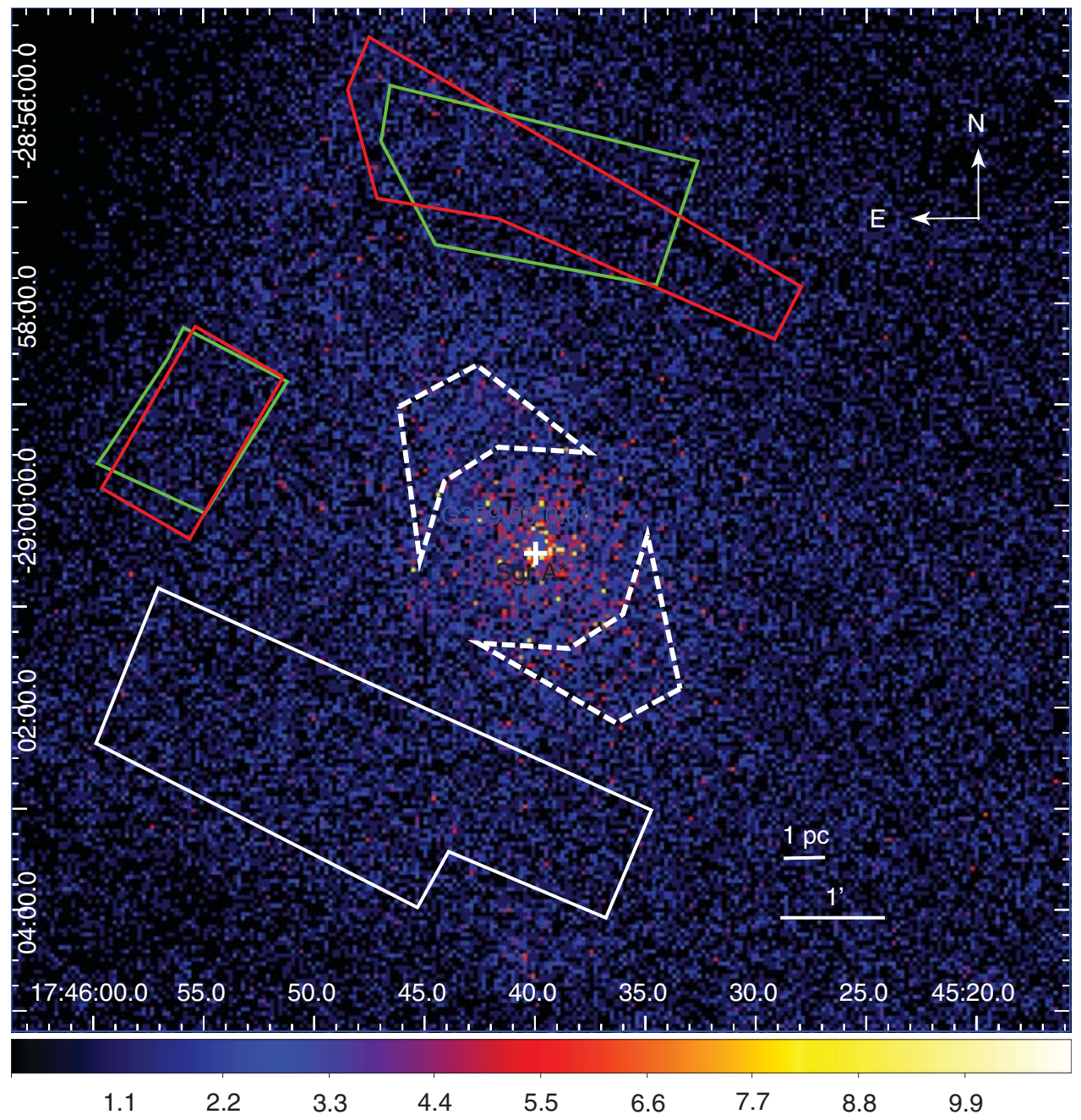

Extended Data Figure 3 Regions used for extraction of background spectra NuSTAR 20-40 keV image. The colour scale shows units of total counts per for NuSTAR observations 30001002001 (red), 30001002003 (white), and 30001002004 (green). The regions used for spectral analysis of the diffuse pixel. The $x$ and $y$ axes indicate coordinates of right ascension and declination, emission are indicated by the dashed lines. These regions are overlaid on the respectively. 


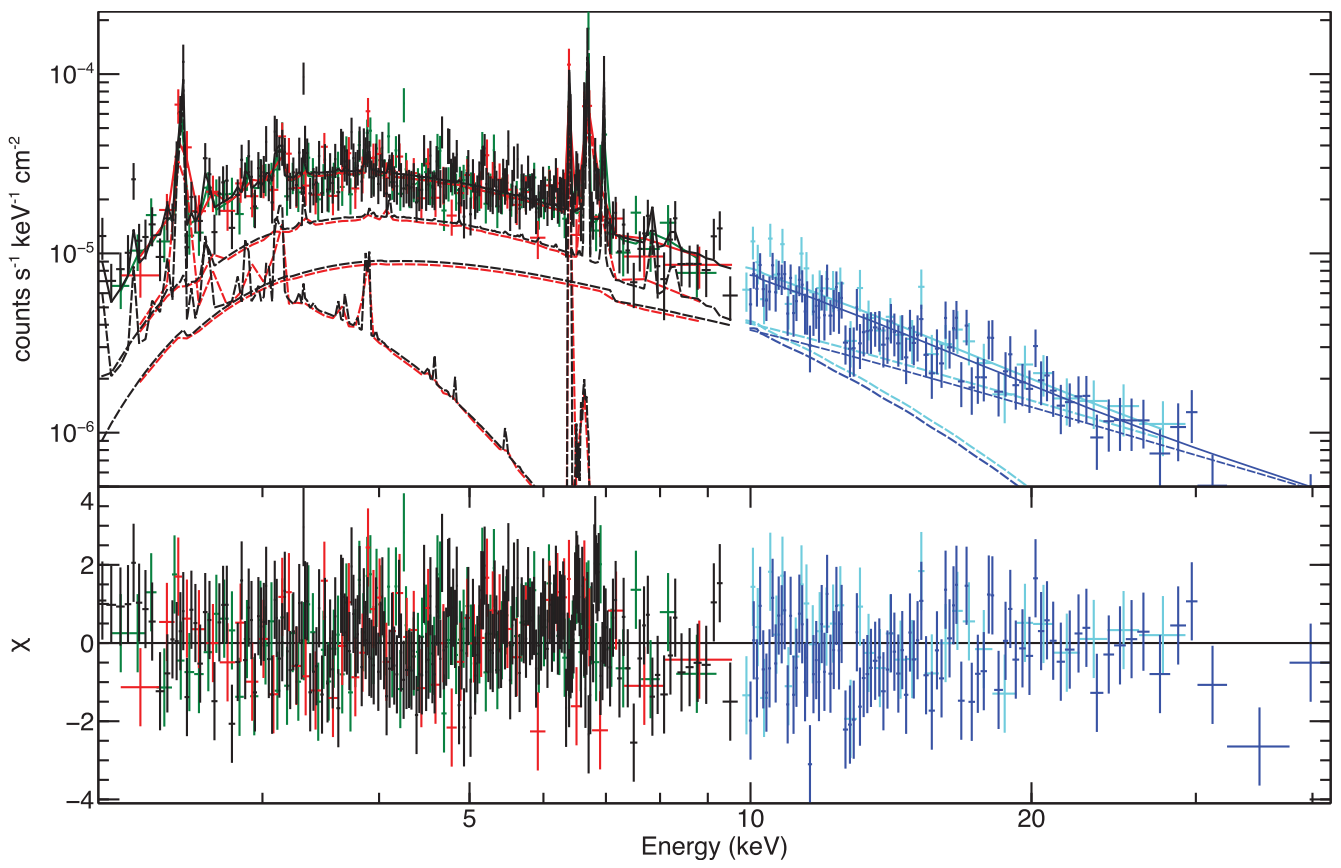

Extended Data Figure $4 \mid$ Unfolded 2-40 keV energy spectrum from the southwest region indicated in Fig. 1. Top panel, the $2-10 \mathrm{keV}$ spectrum is constructed from XMM-Newton data from the PN (black), MOS1 (red), and MOS2 (green) instruments. The $10-40 \mathrm{keV}$ spectrum is constructed from NuSTAR focal plane A (dark blue) and focal plane B (cyan) data. The fit to the data comprises two absorbed thermal plasmas plus an absorbed power-law, multiplied by a separate normalization factor for each data set to account for small calibration differences between instruments. The dashed lines indicate the separate model components. Fit residuals are shown in the lower panel. Full parameters of the spectral model are given in Extended Data Table 2. Error bars, $1 \sigma$ statistical errors. 


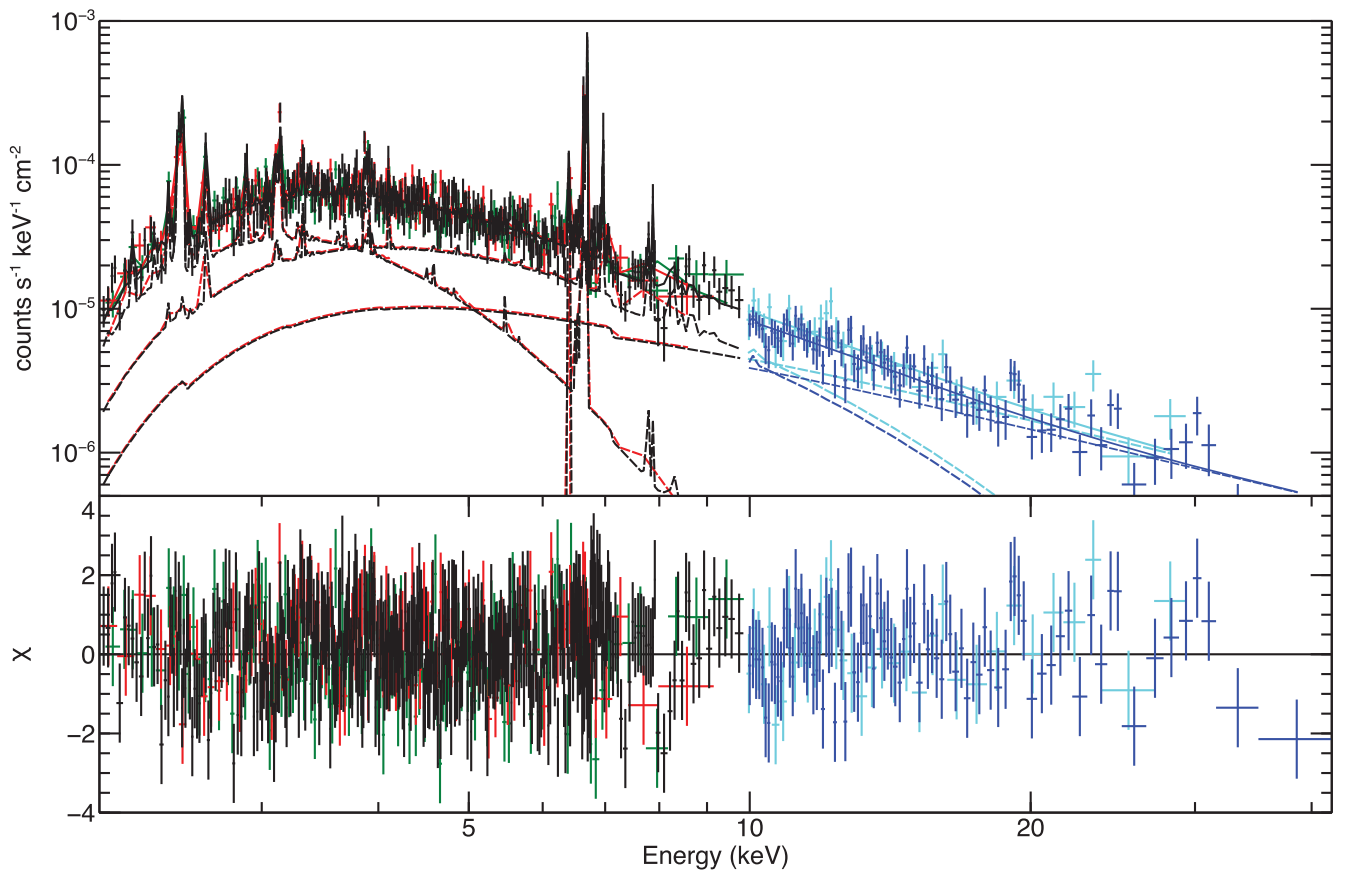

Extended Data Figure $5 \mid$ Unfolded 2-40 keV energy spectrum from the northeast region indicated in Fig. 1. Top panel, the spectrum is composed as in Extended Data Fig. 4. The model fitted to the data comprises an absorbed two-temperature thermal plasma plus a power-law, multiplied by a separate normalization factor for each data set. Fit residuals are shown in the lower panel. Full parameters of the spectral model are given in Extended Data Table 2. Error bars, $1 \sigma$ statistical errors. 


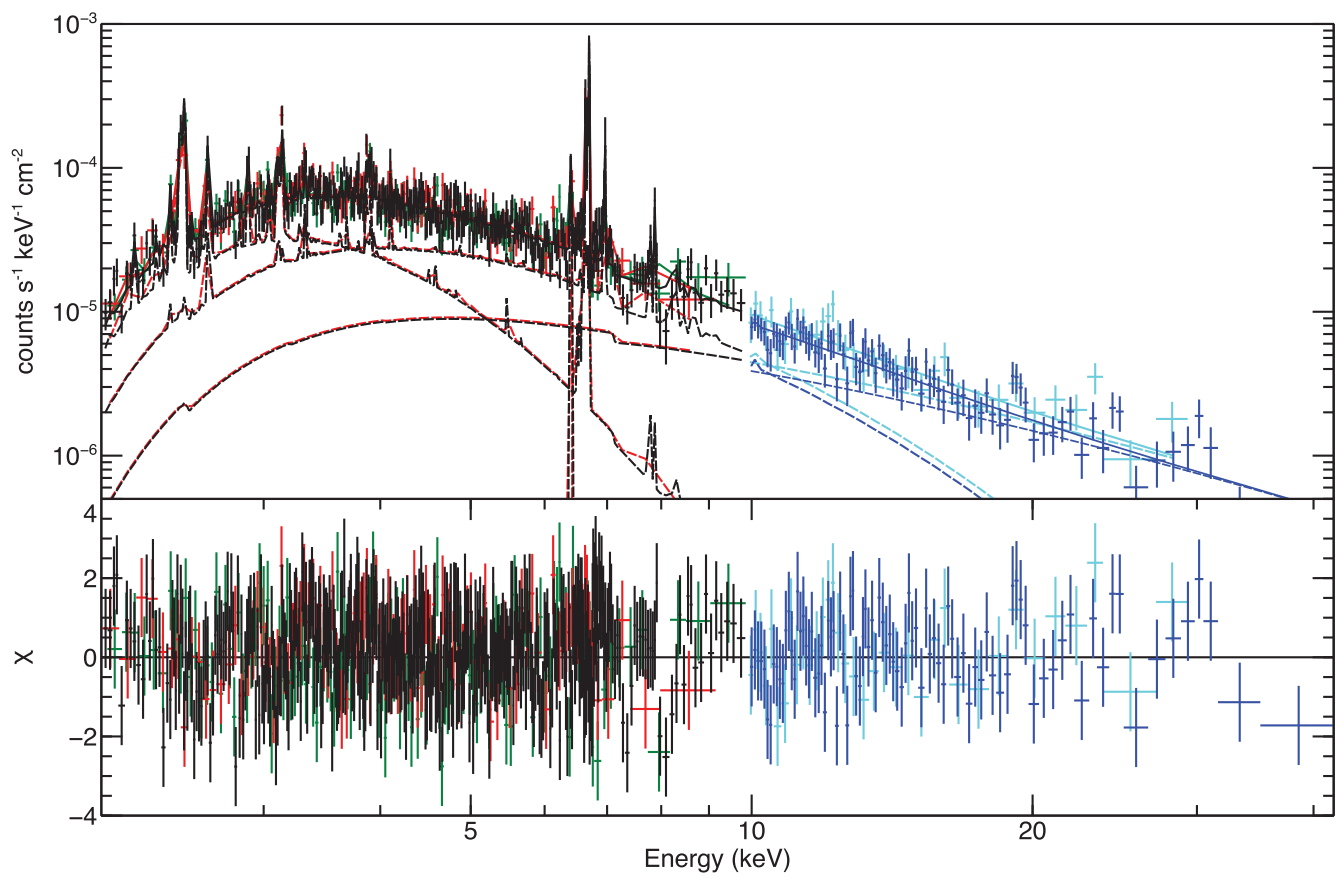

Extended Data Figure 6 | Unfolded 2-40 keV energy spectrum from the northeast region indicated in Fig. 1. The spectrum is composed as in

Extended Data Fig. 4. The model fitted to the data comprises an absorbed twotemperature thermal plasma plus a thermal bremsstrahlung, multiplied by a separate normalization factor for each data set. Fit residuals are shown in the lower panel. Full parameters of the spectral model are given in Extended Data Table 3. Error bars, $1 \sigma$ statistical errors. 
RESEARCH LETTER

Extended Data Table 1 | Best fit 2D Gaussian models

\begin{tabular}{|c|c|c|}
\hline Parameter & G359.95-0.04 & CHXE \\
\hline R.A. (Center, J2000) & $266.4150_{-3}^{\circ}+4.8^{\prime \prime}$ & $266.4172^{\circ}+6.6^{\prime \prime}$ \\
\hline DEC (Center, J2000) & $\begin{array}{l}-29.007245_{-4.1 "}^{\circ}+3.4^{\prime \prime} \\
-{ }^{2}\end{array}$ & $-29.00716_{-7.1 "}^{\circ+8.1 "}$ \\
\hline FWHM [arcseconds] & $1.8_{-0.7}^{+2.3^{-4.1}}$ & $195.8_{-16.8}^{+21.9}$ \\
\hline Amplitude $\left[10^{-3} \mathrm{cts} \mathrm{s}^{-1}\right]$ & 5. $2_{-3.5}^{-0.7}$ & $0.0064_{-0.0017}^{-16.8}$ \\
\hline Ellipticity & - & 0.52 \\
\hline$\theta^{a}$ [degree] & - & 57 \\
\hline
\end{tabular}

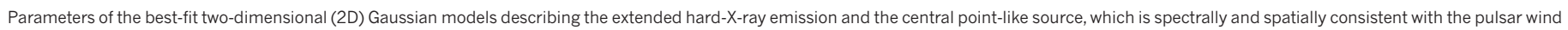
nebula G359.95- 0.04. CHXE is the 20-40 keV extended central hard X-ray emission. The errors quoted are $3 \sigma$ statistical errors. All parameters refer to source models before convolution with the NuSTAR PSF.

${ }^{a}$ The angle $\theta$ is defined with respect to the positive northern axis. 
Extended Data Table 2 | Fits with hard emission modelled as power law

\begin{tabular}{|c|c|c|}
\hline Parameter & Southwest & Northeast \\
\hline $\mathrm{PN}$ norm. ${ }^{a}$ & $1.1_{-0.1}^{+0.1}$ & $1.1_{-0.1}^{+0.1}$ \\
\hline MOS1 and MOS2 norm. & $1.0_{-0.1}^{+0.1}$ & $1.1_{-0.1}^{+0.1}$ \\
\hline NuSTAR FPMA norm. (fixed) & 1.0 & 1.0 \\
\hline NuSTAR FPMB norm. & $1.2_{-0.1}^{+0.1}$ & $1.1_{-0.1}^{+0.1}$ \\
\hline$N_{H}\left[10^{22} \mathrm{~cm}^{-2}\right]$ & $14.1_{-1.3}^{+1.5}$ & $16.4_{-0.8}^{+1.1}$ \\
\hline$\Gamma$ & $1.5_{-0.2}^{+0.3}$ & $1.6_{-0.4}^{+0.8}$ \\
\hline$N_{\Gamma}\left[10^{-4}\right.$ photons $\left.\mathrm{cm}^{-2} \mathrm{~s}^{-1} \mathrm{keV}^{-1}\right]$ & $1.3_{-0.7}^{-0.2}$ & $1.6_{-1.1}^{-0.4}$ \\
\hline$k T_{1}[\mathrm{keV}]$ & $1.0_{-0.4}^{+0.1}$ & $1.1_{-0.1}^{+1.1}$ \\
\hline$N_{k} T_{1}\left[10^{-4}\right.$ photons $\left.\mathrm{cm}^{-2} \mathrm{~s}^{-1} \mathrm{keV}^{-1}\right]$ & $10.8_{-5.0}^{+103}$ & $89.9_{-28.7}^{+5.2 .1}$ \\
\hline$Z_{1}\left[Z_{\odot}\right]^{b}$ & $5.0_{-3.6}^{+-5.0}$ & $2.3_{-0.4}^{-28.7}$ \\
\hline$k T_{2}[\mathrm{keV}]$ & $7.5_{-13}^{+1.6}$ & $5.1_{-0.7}^{+0.4}$ \\
\hline$N_{k} T_{2}\left[10^{-4}\right.$ photons $\left.\mathrm{cm}^{-2} \mathrm{~s}^{-1} \mathrm{keV}^{-1}\right]$ & $9.2_{-1.6}^{+1.3}$ & $17.5_{-5.9}^{+6.7}$ \\
\hline$Z_{2}\left[Z_{\odot}\right]^{c}$ & $1.7^{-1.6}$ & $2.3_{-0.9}^{+0.9}$ \\
\hline Fe K- $\alpha$ eq. width $[\mathrm{eV}]$ & $128_{-31}^{+40}$ & $47_{-16}^{+84.4}$ \\
\hline$\chi^{2} /$ d.o.f. & $1.00(503.4 / 503)$ & $1.05(807.1 / 770)$ \\
\hline$F_{X}(20-40 \mathrm{keV})\left[10^{-13} \mathrm{ergs}^{-2} \mathrm{~s}^{-1}\right]^{d}$ & 7.6 & 8.0 \\
\hline
\end{tabular}

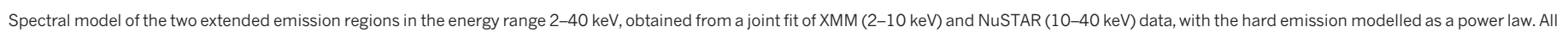

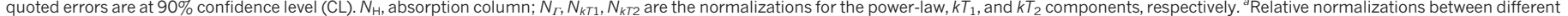

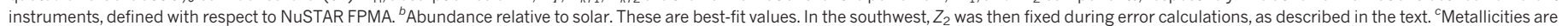
independent (linked) for the two temperature components in the southwest (northeast). ${ }^{d}$ Observed flux. 
RESEARCH LETTER

Extended Data Table 3 | Fits with hard emission modelled as thermal bremsstrahlung

\begin{tabular}{|c|c|c|}
\hline Parameter & Southwest & Northeast \\
\hline PN norm. ${ }^{a}$ & $1.1_{-0.1}^{+0.1}$ & $1.2_{-0.1}^{+0.1}$ \\
\hline MOS1 and MOS2 norm. & $1.1_{-0.1}^{+0.1}$ & $1.2_{-0.1}^{+0.1}$ \\
\hline NuSTAR FPMA norm. (fixed) & $1.0^{-0.1}$ & $1.0^{-0.1}$ \\
\hline NuSTAR FPMB norm. & $1.2_{-0.1}^{+0.1}$ & $1.2_{-0}^{+0.1}$ \\
\hline$N_{H}\left[10^{22} \mathrm{~cm}^{-2}\right]$ & $13.4_{-1.3}^{+1.6}$ & $16.4_{-0.8}^{+1.2}$ \\
\hline$k T_{\text {bremss }}[\mathrm{keV}]$ & $58_{-23}^{+127}$ & $66_{-30}^{+203^{\circ}}$ \\
\hline$N_{\text {bremss }}\left[10^{-4}\right.$ photons $\left.\mathrm{cm}^{-2} \mathrm{~s}^{-1} \mathrm{keV}^{-1}\right]$ & $1.8_{-0.4}^{+0.4}$ & $1.9_{-0.3}^{+30}$ \\
\hline$k T_{1}[\mathrm{keV}]$ & $1.0_{-0.3}^{+0.4}$ & $1.1+0.1$ \\
\hline$N_{k} T_{1}\left[10^{-4}\right.$ photons $\left.\mathrm{cm}^{-2} \mathrm{~s}^{-1} \mathrm{keV}^{-1}\right]$ & $9.5_{-2.0}^{+30}$ & $93.2_{-22.2}^{+55.0}$ \\
\hline$Z_{1}\left[Z_{\odot}\right]^{b}$ & $5.0_{-3.2}^{+--}$ & $2.2_{-0.3}^{+0.4}$ \\
\hline$k T_{2}[\mathrm{keV}]$ & $7.2_{-1.3}^{+1.4}$ & $5.0_{-0.7}^{+0.9}$ \\
\hline$N_{k T_{2}}\left[10^{-4}\right.$ photons $\left.\mathrm{cm}^{-2} \mathrm{~s}^{-1} \mathrm{keV}^{-1}\right]$ & $8.8_{-1.8}^{+2.0}$ & $18.0_{-4.8}^{+6.1}$ \\
\hline$Z_{2}\left[Z_{\odot}\right]^{c}$ & 1.6 & $2.2_{-0.3}^{+0.4}$ \\
\hline Fe K- $\alpha$ eq. width [eV] & $123_{-46}^{+90}$ & $46_{-13}^{+12}$ \\
\hline$\chi^{2} /$ d.o.f. & $1.00(501.6 / 503)$ & $1.05(807.1 / 770)$ \\
\hline$F_{X}(20-40 \mathrm{keV})\left[10^{-13} \mathrm{ergs} \mathrm{cm}^{-2} \mathrm{~s}^{-1}\right]^{d}$ & 7.3 & 8.0 \\
\hline
\end{tabular}

Model and footnotes as in Extended Data Table 2, but with the hard emission modelled as a thermal bremsstrahlung. $N_{\text {bremss }}$ is the normalization of the high-temperature bremsstrahlung component. 